\title{
EFEITO DA PROFUNDIDADE DE SEMEADURA E DE RODAS COMPACTADORAS SUBMETIDAS A CARGAS VERTICAIS NA TEMPERATURA E NO TEOR DE ÁGUA DO SOLO DURANTE A GERMINAÇÃO DE SEMENTES DE MILHO ${ }^{1}$
}

\author{
Effect of sowing depth and press wheels submitted to vertical loads on soil temperature and \\ moisture contents during corn seed germination
}

\author{
Rouverson Pereira da Silva 2 , José Eduardo Coráa ${ }^{3}$ Alberto Carvalho Filho ${ }^{4}$, \\ Carlos Eduardo Angeli Furlani5, Afonso Lopes 6
}

\begin{abstract}
RESUMO
As rodas compactadoras das semadoras-adubadoras têm como principal função, propiciar o bom contato entre o solo e a semente para garantir a germinação das sementes e a emergência das plântulas. Objetivou-se, neste trabalho, estudar a influência de três modelos de rodas compactadoras, três profundidades de semeadura e três níveis de carga sobre a roda compactadora sob a temperatura e o teor de água do solo. O trabalho foi desenvolvido na pista de ensaios de semeadura, localiza em Uberaba, MG, em Latossolo Vermelho distrófico, utilizando-se o delineamento experimental de parcelas sub-subdivididas, com 27 tratamentos e quatro repetições. As rodas com maior área de contato com o solo proporcionaram maiores valores do teor médio de água e da temperatura do solo. O nível de carga sobre a roda compactadora não afetou a temperatura e o teor de água do solo.
\end{abstract}

Termos para indexação: Teor de água do solo, temperatura do solo, emergência.

\begin{abstract}
Press wheels were designed to improve soil-seed contact, thus promoting good seed germination and emergence of plantules. The present investigation was aimed at studying the influence of three models of press wheel, three sowing depths and three load levels on soil temperature and moisture contents. The experiment was carried out in Uberaba, State of Minas Gerais, Brazil, in splitplot desing, 27 treatments and four repetitions. Results have shown that press wheels with bigger soil contact area provide the highest values of average soil moisture an temperature. However, load level on the press has shown no effect on soil temperature and moisture contents.
\end{abstract}

Index terms: Soil moisture, soil temperature, emergence.

(Recebido em 9 de março de 2006 e aprovado em 18 de abril de 2007)

\section{INTRODUÇÃO}

Nas culturas anuais a operação de semeadura se reveste de grande importância, pois possíveis erros durante essa etapa só poderão ser detectados após a emergência, conduzindo a perdas significativas de produção. As rodas compactadoras são dispositivos que melhoram o contato solo-semente por meio da aplicação de pressão exercida lateralmente e sobre a linha de semeadura, provocando leve compactação e deixando que o solo, diretamente sobre as sementes, fique solto o suficiente de forma a envolvê- las completamente, sem no entanto permitir a formação de crostas, garantindo a emergência mais fácil das plântulas.

Outro fator a ser considerado em uma operação de semeadura é a profundidade de deposição das sementes, que pode afetar sua germinação, sendo condicionada pela temperatura, teor de água e tipo de solo, dentre outros fatores. A semente deve ser depositada a uma profundidade que permita um bom contato com o solo úmido, resultando em uma boa germinação.

De acordo com Ortolani et al. (1986), citado por Cortez et al. (2006), durante a germinação, três fatores são

\footnotetext{
Parte da Tese de Doutorado do $1^{\circ}$ autor junto à FCAV/UNESP

${ }^{2}$ Engenheiro Agrícola Doutor, Professor Assistente - Departamento de Engenharia Rural/DER - Universidade Estadual Paulista/UNESP - Via de Acesso Prof. Paulo Donato Castellane, s/n - 14884-900 - Jaboticabal, SP - rouverson@fcav.unesp.br - Bolsista de Produtividade em Pesquisa do CNPq ${ }^{3}$ Engenheiro Agrônomo, Doutor, Professor Adjunto - Departamento de Solos e Adubos/DSA - Universidade Estadual Paulista/UNESP - Via de Acesso Prof. Paulo Donato Castellane, s/n - 14884-900 - Jaboticabal, SP - cora@fcav.unesp.br

${ }^{4}$ Engenheiro Agrônomo, Doutor, Professor - Faculdades Associadas de Uberaba/FAZU - Avenida do Tutuna, 720, Tutunas - 38061-500 - Uberaba, MG alberto@fazu.br

${ }^{5}$ Engenheiro Agrônomo, Doutor, Professor Adjunto - Departamento de Engenharia Rural/DER - Universidade Estadual Paulista/UNESP - Via de Acesso Prof. Paulo Donato Castellane, s/n - 14884-900 - Jaboticabal, SP - furlani@fcav.unesp.br - Bolsista de Produtividade em Pesquisa do CNPq Engenheiro Agrícola, Doutor, Professor Adjunto - Departamento de Engenharia Rural/DER - Universidade Estadual Paulista/UNESP - Via de Acesso Prof. Paulo Donato Castellane, s/n - 14884-900 - Jaboticabal, SP - afonso@fcav.unesp.br
} 
fundamentais e devem estar adequadamente presentes: calor; teor de água e oxigênio. Para a emergência, a profundidade de semeadura representa um quarto fator fundamental, pois mesmo havendo boa germinação, pode impedir uma boa emergência.

As rodas compactadoras têm a função de aumentar o contato das sementes com o solo, para melhorar a absorção de água e garantir melhor germinação (PACHECO, 1994). Stefanutti (1979), citado por Silva et al. (2006), trabalhando com um único modelo de roda, mudando apenas as cargas de compactação, constatou a alteração de valores de densidade e temperatura do solo em profundidade, comprovando que a modificação do comportamento físico proporcionado às sementes e plântulas é provocada pela regulagem de carga da roda compactadora sobre o solo.

Silva (1990) realizou um estudo para verificar a influência de quatro diferentes modelos de rodas compactadoras em alguns parâmetros físicos do solo e também no desenvolvimento das plantas na cultura do feijão, utilizando três níveis de compactação. Seus resultados mostraram que existe um comportamento distinto entre os tipos de rodas e cargas de compactação em relação ao desenvolvimento de plantas, à produção de grãos e à alteração nas características físicas do solo.

Conduziu-se, este trabalho, para estudar a influência de três modelos de rodas compactadoras, três profundidades de semeadura e três níveis de carga sobre as rodas compactadoras na temperatura e teor de água do solo durante o período de germinação de sementes de milho, em uma pista de ensaios projetada para essa finalidade.

\section{MATERIAL E MÉTODOS}

O trabalho foi desenvolvido na Área Experimental da FAZU - Faculdades Associadas de Uberaba localizada no município de Uberaba, MG, cujas coordenadas geográficas são: Latitude: 1944'13" S, Longitude: 4757'27" W e Altitude de $780 \mathrm{~m}$. O solo da área foi classificado como Latossolo Vermelho distrófico, segundo o Sistema Brasileiro de Classificação de Solos (EMBRAPA, 1999).

A pista é constituída de sete canais de solo nivelados, delimitados por trilhos, sobre os quais desliza um trole acionado por motor elétrico. Para realização dos ensaios foram escolhidos três modelos de rodas, classificadas de acordo com a ASAE (1995) em:

- roda 1: roda duplo-angulada, constituída por duas rodas cilíndricas em "V", revestidas com borracha maciça, normalmente usada para plantio direto;
- roda 2: roda convexa larga, revestida com borracha deformável;

- roda 3: roda dupla com nervura simples em "V", constituída por duas rodas cilíndricas revestidas de borracha maciça, com nervura central.

Os tratamentos foram constituídos pela combinação dos três modelos de rodas compactadoras com três níveis de carga $(50,100$ e $150 \mathrm{~N})$ e três profundidades de semeadura $(3,5$ e $7 \mathrm{~cm})$, totalizando 27 tratamentos (Tabela 1). Foi utilizada a cultivar de milho híbrido simples, Aventis A2288, superprecoce, com pureza mínima de $98 \%$ e germinação mínima de $85 \%$, que possui características adequadas para exploração na zona macro-agroecológica do Cerrado do Brasil Central, conforme classificação de Monteiro et al. (1992).

O delineamento estatístico adotado foi de blocos casualizados em sub-subparcelas, com quatro repetições. As parcelas foram instaladas ao longo das linhas de semeadura, sendo caracterizadas pelo modelo da roda compactadora. As subparcelas foram compostas pelas profundidades de semeadura e as sub-subparcelas pelos níveis de carga.

A aplicação dos tratamentos obedeceu às seguintes etapas:

- o comprimento das parcelas foi estabelecido em 2 metros em toda a largura dos canais, com três fileiras espaçadas de $0,75 \mathrm{~m}$, nas quais foram depositadas 5 sementes.m ${ }^{-1}$, resultando em 70.000 plantas.ha ${ }^{-1}$;

- a semeadura foi realizada de forma manual, abrindo-se pequenas covas com o auxílio de cilindros de madeira posicionados sobre uma régua de madeira com furos no espaçamento desejado. Os cilindros, com comprimento nas profundidades desejadas e diâmetro de $15 \mathrm{~mm}$, foram comprimidos contra o solo até encostar na régua;

- logo após a semeadura as rodas foram fixadas ao suporte e esse à face vertical do trole porta-ferramentas, sendo então posicionadas de modo que a linha de semeadura ficasse exatamente no centro do plano vertical das rodas compactadoras;

- as rodas foram posicionadas no centro das entrefaixas e as cargas estáticas correspondentes a cada tratamento foram colocadas cuidadosamente sobre o eixo vertical das rodas, evitando-se impactos no solo.

- o trole foi acionado até início da próxima entrefaixa, quando as cargas foram então alteradas de acordo com o tratamento a ser aplicado. Esse procedimento foi repetido até o fim do canal, quando o trole foi mudado de canal. 
Tabela 1 - Relação e designação dos tratamentos.

\begin{tabular}{|c|c|c|c|}
\hline Tratamentos & Roda & Profundidade $(\mathrm{cm})$ & Carga $(\mathrm{N})$ \\
\hline R1P1C1: & Roda 1 & 3 & 50 \\
\hline R1P1C2: & Roda 1 & 3 & 100 \\
\hline R1P1C3: & Roda 1 & 3 & 150 \\
\hline R1P2C1: & Roda 1 & 5 & 50 \\
\hline R1P2C2: & Roda 1 & 5 & 100 \\
\hline R1P2C3: & Roda 1 & 5 & 150 \\
\hline R1P3C1: & Roda 1 & 7 & 50 \\
\hline R1P3C2: & Roda 1 & 7 & 100 \\
\hline R1P3C3: & Roda 1 & 7 & 150 \\
\hline R2P1C1: & Roda 2 & 3 & 50 \\
\hline R2P1C2: & Roda 2 & 3 & 100 \\
\hline R2P1C3: & Roda 2 & 3 & 150 \\
\hline $\mathrm{R} 2 \mathrm{P} 2 \mathrm{C} 1 \mathrm{:}$ & Roda 2 & 5 & 50 \\
\hline $\mathrm{R} 2 \mathrm{P} 2 \mathrm{C} 2 \mathrm{:}$ & Roda 2 & 5 & 100 \\
\hline R2P2C3: & Roda 2 & 5 & 150 \\
\hline R2P3C1: & Roda 2 & 7 & 50 \\
\hline R2P3C2: & Roda 2 & 7 & 100 \\
\hline R2P3C3: & Roda 2 & 7 & 150 \\
\hline R3P1C1: & Roda 3 & 3 & 50 \\
\hline R3P1C2: & Roda 3 & 3 & 100 \\
\hline R3P1C3: & Roda 3 & 3 & 150 \\
\hline R3P2C1: & Roda 3 & 5 & 50 \\
\hline $\mathrm{R} 3 \mathrm{P} 2 \mathrm{C} 2 \mathrm{:}$ & Roda 3 & 5 & 100 \\
\hline R3P2C3: & Roda 3 & 5 & 150 \\
\hline R3P3C1: & Roda 3 & 7 & 50 \\
\hline R3P3C2: & Roda 3 & 7 & 100 \\
\hline R3P3C3: & Roda 3 & 7 & 150 \\
\hline
\end{tabular}

As determinações do teor de água e temperatura do solo foram realizadas diariamente, durante a fase de germinação das sementes e emergência das plântulas, no período compreendido entre a semeadura e o desbaste. A avaliação do teor de água do solo foi feita pelo método gravimétrico (EMBRAPA, 1997). Para essa determinação foram retiradas quatro amostras em cada tratamento, com auxílio de um calador, na profundidade de semeadura de cada parcela, sempre às 09:00 horas.

A temperatura do solo foi determinada diariamente às 9 horas, em cada tratamento, nas profundidades de semeadura utilizando-se geotermômetros de mercúrio, com resolução de $0,2{ }^{\circ} \mathrm{C}$, instalados entre duas plantas consecutivas na linha de semeadura, em cada sub-sub-parcela. Os resultados obtidos foram tabulados e submetidos à análise de variância, utilizando-se o programa computacional SANEST. Quando o valor do teste F mostrou-se significativo a 5\% de probabilidade, aplicouse o teste de Tukey para comparação das médias. Para as interações significativas realizou-se o desdobramento da análise de variância, considerando-se os fatores profundidade e carga isoladamente. 


\section{RESULTADOS E DISCUSSÃO}

Para respaldar as discussões são apresentadas as análises de variância para os valores médios de temperatura e de teor de água do solo, obtidos durante a fase de germinação e emergência da cultura, conforme a Tabela 2 .

A análise de variância demonstrou que o teor de água do solo foi afetado pelo modelo de roda compactadora (R) e pelo nível de carga aplicado (C). As interações entre modelo de roda e nível de carga $(\mathrm{RxC})$ e entre profundidade de semeadura e nível de carga (PxC), também foram significativas (Tabela 2). O teor de água do solo não diferiu estatisticamente para os tratamentos com as rodas 2 e 3 , que proporcionaram maiores valores para o teor médio de água do solo quando comparadas com a roda 1 , concordando com Silva (1990), que também não encontrou diferenças significativas para as médias de teor de água do solo proporcionado pelo uso dos modelos de roda 2 e 3 .
As rodas 2 e 3, por apresentarem maior área de contato com o solo proporcionaram maiores valores do teor médio de água e da temperatura do solo, no período de emergência do milho.

Com relação ao nível de carga aplicado, observa-se que a aplicação da carga de $100 \mathrm{~N}$ (C2) proporcionou um valor mais elevado para o teor de água do solo do que a carga de $150 \mathrm{~N}$ (C3) (Tabela 2). Esses resultados divergem dos resultados obtidos por Silva (1990), que encontrou maiores teores de água do solo para tratamentos que receberam maiores cargas de compactação.

Por outro lado, a temperatura do solo foi afetada pelo modelo de roda, pela profundidade de semeadura e pelas interações entre modelo de roda e profundidade $(\mathrm{RxP})$, modelo de roda e nível de carga $(\mathrm{RxC})$ e entre os três fatores ( $\mathrm{RxPxC}$ ) (Tabela 2). Entretanto é conveniente ressaltar que todos os fatores analisados proporcionaram

Tabela 2 - Síntese da análise de variância e do teste de médias para os valores médios do teor de água (U) e temperatura do solo (T), no período de germinação e emergência das plântulas.

\begin{tabular}{|c|c|c|}
\hline Fator & $\mathrm{U}(\%)$ & $\mathrm{T}\left({ }^{\circ} \mathrm{C}\right)$ \\
\hline Roda 1 & $12,57 \mathrm{~b}$ & 28,46 c \\
\hline Roda 2 & $13,96 \mathrm{a}$ & $28,97 \mathrm{a}$ \\
\hline Roda 3 & $14,12 \mathrm{a}$ & $28,65 \mathrm{~b}$ \\
\hline Profundidade 1 & $13,48 \mathrm{a}$ & 29,03 a \\
\hline Profundidade 2 & $13,51 \mathrm{a}$ & $28,72 \mathrm{~b}$ \\
\hline Profundidade 3 & $13,66 \mathrm{a}$ & $28,34 \mathrm{c}$ \\
\hline Carga 1 & $13,60 \mathrm{ab}$ & $28,66 \mathrm{a}$ \\
\hline Carga 2 & $13,66 \mathrm{a}$ & $28,71 \mathrm{a}$ \\
\hline Carga 3 & $13,38 \mathrm{~b}$ & $28,71 \mathrm{a}$ \\
\hline \multicolumn{3}{|l|}{ Teste F } \\
\hline Rodas (R) & $231,42^{*}$ & $3,96^{*}$ \\
\hline Profundidades (P) & $1,36^{\mathrm{NS}}$ & $109,22 *$ \\
\hline $\mathrm{R} \times \mathrm{P}$ & $2,05^{\mathrm{NS}}$ & $6,56^{*}$ \\
\hline Carga & $4,16^{*}$ & $1,90^{\mathrm{NS}}$ \\
\hline $\mathrm{R} \times \mathrm{C}$ & $6,06^{*}$ & $7,79 *$ \\
\hline P X C & $3,84 *$ & $1,74^{\mathrm{NS}}$ \\
\hline R X P X C & $1,88^{\mathrm{NS}}$ & $7,95^{*}$ \\
\hline C.V. Roda (\%) & 0,82 & 0,90 \\
\hline C.V. Profundidade (\%) & 2,05 & 0,40 \\
\hline C.V. Carga (\%) & 3,19 & 0,41 \\
\hline
\end{tabular}

*: significativo a $5 \%$ de probabilidade; NS: não significativo; CV: coeficiente de variação. 
temperaturas médias situadas no intervalo entre 20 e $30^{\circ} \mathrm{C}$, o que de acordo com por Popinigis (1985), é considerado ideal para que ocorra a germinação.

O maior valor de temperatura média do solo na região da semente foi observado usando-se o modelo de roda 2, quando comparado aos valores obtidos com a utilização dos modelos 3 e 1 (Tabela 2). Isso pode ser explicado pela geometria da roda 2 , pois rodas convexas exercem pressão de forma mais direta sobre a linha de semeadura, enquanto que nas rodas em "V" a pressão é exercida mais lateralmente (SILVA et al., 2005). Silva (1990), também encontrou para esse modelo de roda, um comportamento diferente, com registro de temperatura mais elevada que as demais. Os resultados permitem observar que, sementes colocadas a profundidade de $3 \mathrm{~cm}$ (P1) encontraram um ambiente com temperatura do solo mais alta do que aquelas semeadas em profundidades de $5(\mathrm{P} 2)$ e $7 \mathrm{~cm}(\mathrm{P} 3)$.

Com relação à carga de compactação os resultados obtidos são condizentes com os verificados por Silva (1990), que também não identificou diferenças significativas entre os valores de temperatura para tratamentos com diferentes cargas de compactação (Tabela 2).

\section{Interação Roda x Profundidade}

O teste de médias indicou que, em todas as profundidades estudadas, o modelo de roda 2 proporcionou maiores valores de temperatura do solo (Tabela 3). Para a profundidade de $3 \mathrm{~cm}$ (P1) não houve diferença significativa entre os modelos de roda 1 e 3 , enquanto que para as profundidades de $5(\mathrm{P} 2)$ e $7 \mathrm{~cm}(\mathrm{P} 3)$, os tratamentos com roda 1 , apresentaram as menores médias para a temperatura do solo (Tabela 3 ).

\section{Interação Roda x Carga}

Quando analisado o efeito dos modelos de roda em cada nível de carga (Tabela 4), observa-se que o teor de água no solo foi menor para a roda 1 em todos os níveis de carga avaliados. Para os níveis de carga $1(50 \mathrm{~N})$ e $2(100 \mathrm{~N})$ o teor de água no solo foi estatisticamente semelhante para os modelos de rodas 1 e 2, enquanto que para o nível de carga $3(150 \mathrm{~N})$ os tratamentos diferenciaram entre si, apresentando maiores teores de água no solo com as rodas 3, 2 e 1, nessa sequiência.

Em relação à temperatura do solo, a roda 2 foi a que proporcionou as maiores médias encontradas dentro dos níveis de carga 1 e 2 (Tabela 4), o que pode ser justificado pelo fato dessas rodas apresentarem maior área de contato com o solo, possibilitando o aumento do contato das sementes com o solo (PACHECO, 1994, citado por SILVA et al., 2005).

\section{Interação Profundidade x Carga}

O efeito da profundidade dentro de cada nível de carga apresentou diferença significativa somente para a carga de $150 \mathrm{~N}$ (C3), com os maiores valores do teor de água do solo ocorrendo para as profundidades de 7 (P3) e $5 \mathrm{~cm}$ (P2) (Tabela 5). Isso pode ser explicado pelo fato de que rodas com maior área de contato com o solo proporcionam maiores valores do teor médio de água do solo e da temperatura do solo.

\section{Interação Roda x Profundidade x Carga}

Em todas as combinações, o modelo de roda 2 foi o que proporcionou as maiores médias de temperatura do solo (Tabela 6). Nas interações com P2C2, P2C3 e P3C3 os valores da temperatura não diferiram entre si para os modelos de roda 2 e 3 .

Tabela 3 - Médias dos valores de temperatura do solo (T) para estudo do efeito do fator roda dentro do fator profundidade.

\begin{tabular}{cll}
\hline Efeito & Fator & $\mathrm{T}\left({ }^{\circ} \mathrm{C}\right)$ \\
\hline \multirow{2}{*}{ Roda dentro de Profundidade 1 } & Roda 1 & $28,77 \mathrm{~b}$ \\
& Roda 2 & $29,48 \mathrm{a}$ \\
& Roda 3 & $28,83 \mathrm{~b}$ \\
\hline Roda dentro de Profundidade 2 & Roda 1 & $28,49 \mathrm{c}$ \\
& Roda 2 & $28,89 \mathrm{a}$ \\
Roda dentro de Profundidade 3 & Roda 3 & $28,77 \mathrm{~b}$ \\
\hline
\end{tabular}

Em cada coluna, para cada efeito, médias seguidas de mesma letra não diferem entre si, pelo teste de Tukey, a 5\% de probabilidade. 
Tabela 4 - Médias dos valores do teor de água (U) e da temperatura do solo (T) para estudo do efeito do fator roda dentro do fator carga.

\begin{tabular}{clll}
\hline Efeito & Fator & U $(\%)$ & T $\left({ }^{\circ} \mathrm{C}\right)$ \\
\hline \multirow{2}{*}{ Roda dentro de Carga 1 } & Roda 1 & $12,37 \mathrm{~b}$ & $28,46 \mathrm{~b}$ \\
& Roda 2 & $14,25 \mathrm{a}$ & $29,01 \mathrm{a}$ \\
& Roda 3 & $14,18 \mathrm{a}$ & $28,52 \mathrm{~b}$ \\
\hline \multirow{2}{*}{ Roda dentro de Carga 2 } & Roda 1 & $12,70 \mathrm{~b}$ & $28,40 \mathrm{c}$ \\
& Roda 2 & $14,20 \mathrm{a}$ & $29,00 \mathrm{a}$ \\
Roda dentro de Carga 3 & Roda 3 & $14,09 \mathrm{a}$ & $28,72 \mathrm{~b}$ \\
\hline
\end{tabular}

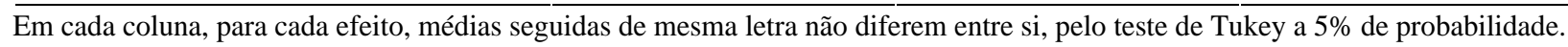

Tabela 5 - Médias do teor de água do solo (U) para estudo do efeito do fator profundidade dentro do fator carga.

\begin{tabular}{ccl}
\hline Efeito & Fator & $\mathrm{U}(\%)$ \\
\hline & $\mathrm{P} 1$ & $13,75 \mathrm{a}$ \\
Profundidade dentro de Carga 1 & $\mathrm{P} 2$ & $13,58 \mathrm{a}$ \\
& $\mathrm{P} 3$ & $13,47 \mathrm{a}$ \\
\hline Profundidade dentro de Carga 2 & $\mathrm{P} 1$ & $13,65 \mathrm{a}$ \\
& $\mathrm{P} 2$ & $13,53 \mathrm{a}$ \\
Profundidade dentro de Carga 3 & $\mathrm{P} 3$ & $13,81 \mathrm{a}$ \\
\hline
\end{tabular}

Em cada coluna, para cada fator, médias seguidas de mesma letra não diferem entre si, pelo teste de Tukey a 5\% de probabilidade.

Quando verificado o efeito da profundidade de semeadura dentro da combinação de roda e carga, verificou-se que as maiores médias de temperatura do solo ocorreram para a profundidade de $3 \mathrm{~cm}$ (P1), enquanto as menores médias ocorreram para a profundidade de $7 \mathrm{~cm}$ (P3) (Tabela 7).

A análise dos resultados obtidos para as variáveis do solo revelou que o nível de carga aplicado ao solo pelas rodas compactadoras, a geometria das rodas e a profundidade de semeadura podem provocar pequenas alterações no ambiente físico do solo, que, apesar de serem de difícil detecção, ajudam na compreensão da relação máquina-solo. Essas alterações foram também constatadas por Silva et al. (2005), que verificaram, para dois modelos de rodas compactadoras em "V", a ocorrência de compactação lateral do solo na linha de semeadura; enquanto para o modelo de roda convexa a pressão foi exercida de forma direta sobre a linha de semeadura, observando ainda que, para um dos modelos de roda compactadora em "V", cargas verticais no intervalo de 75 a $325 \mathrm{~N}$ provocaram a elevação central do solo na linha de semeadura. Entretanto, para cargas acima de $325 \mathrm{~N}$, houve a redução da elevação central, com ocorrência de compactação direta sobre a linha de semeadura.

As rodas com maior área de contato com o solo (roda 2, 3 e 1, na seqüência) proporcionaram maiores valores do teor médio de água e da temperatura do solo. Comportamento semelhante ocorre quando se avalia o 
Tabela 6 - Médias dos valores de temperatura do solo (T) para estudo do efeito do fator roda dentro dos fatores profundidade e carga.

\begin{tabular}{|c|c|c|}
\hline Efeito & Fator & $\mathrm{T}\left({ }^{\circ} \mathrm{C}\right)$ \\
\hline \multirow{3}{*}{ Roda dentro de Profundidade 1 e Carga 1} & Roda 1 & $28,92 \mathrm{~b}$ \\
\hline & Roda 2 & $29,38 \mathrm{a}$ \\
\hline & Roda 3 & $28,65 \mathrm{c}$ \\
\hline \multirow{3}{*}{ Roda dentro de Profundidade 1 e Carga 2} & Roda 1 & $28,50 \mathrm{c}$ \\
\hline & Roda 2 & $29,55 \mathrm{a}$ \\
\hline & Roda 3 & $29,05 \mathrm{~b}$ \\
\hline \multirow{3}{*}{ Roda dentro de Profundidade 1 e Carga 3} & Roda 1 & $28,88 \mathrm{~b}$ \\
\hline & Roda 2 & $29,52 \mathrm{a}$ \\
\hline & Roda 3 & $28,80 \mathrm{~b}$ \\
\hline \multirow{3}{*}{ Roda dentro de Profundidade 2 e Carga 1} & Roda 1 & $28,45 \mathrm{c}$ \\
\hline & Roda 2 & $29,08 \mathrm{a}$ \\
\hline & Roda 3 & $28,68 \mathrm{~b}$ \\
\hline \multirow{3}{*}{ Roda dentro de Profundidade 2 e Carga 2} & Roda 1 & $28,52 \mathrm{~b}$ \\
\hline & Roda 2 & $28,90 \mathrm{a}$ \\
\hline & Roda 3 & $28,78 \mathrm{a}$ \\
\hline \multirow{3}{*}{ Roda dentro de Profundidade 2 e Carga 3} & Roda 1 & $28,50 \mathrm{~b}$ \\
\hline & Roda 2 & $28,70 \mathrm{a}$ \\
\hline & Roda 3 & $28,85 \mathrm{a}$ \\
\hline \multirow{3}{*}{ Roda dentro de Profundidade 3 e Carga 1} & Roda 1 & $28,00 \mathrm{c}$ \\
\hline & Roda 2 & $28,58 \mathrm{a}$ \\
\hline & Roda 3 & $28,25 \mathrm{~b}$ \\
\hline \multirow{3}{*}{ Roda dentro de Profundidade 3 e Carga 2} & Roda 1 & $28,18 \mathrm{~b}$ \\
\hline & Roda 2 & $28,55 \mathrm{a}$ \\
\hline & Roda 3 & $28,32 \mathrm{~b}$ \\
\hline \multirow{3}{*}{ Roda dentro de Profundidade 3 e Carga 3} & Roda 1 & $28,20 \mathrm{~b}$ \\
\hline & Roda 2 & $28,48 \mathrm{a}$ \\
\hline & Roda 3 & $28,50 \mathrm{a}$ \\
\hline
\end{tabular}

Em cada coluna, para cada fator, médias seguidas de mesma letra não diferem entre si, pelo teste de Tukey a $5 \%$ de probabilidade.

efeito do modelo de roda em cada carga e, para a variável temperatura do solo quando avaliados os modelos de roda em cada profundidade e na combinação de profundidade e carga. De acordo com Rainbow (2000), diferentes modelos de rodas compactadoras apresentam considerável influência sobre o ambiente do solo em torno da semente, sendo essa influência dependente do nível de pressão e do desenho da roda. 
Tabela 7 - Médias dos valores de temperatura do solo (T) para estudo do efeito do fator profundidade dentro dos fatores roda e carga.

\begin{tabular}{|c|c|c|}
\hline Efeito & Fator & $\mathrm{T}\left({ }^{\mathrm{o}} \mathrm{C}\right)$ \\
\hline \multirow{3}{*}{ Profundidade dentro de Roda 1 e Carga 1} & P 1 & $29,92 \mathrm{a}$ \\
\hline & $\mathrm{P} 2$ & $28,45 \mathrm{~b}$ \\
\hline & P 3 & $28,00 \mathrm{c}$ \\
\hline \multirow{3}{*}{ Profundidade dentro de Roda 1 e Carga 2} & P 1 & $28,50 \mathrm{a}$ \\
\hline & P 2 & $28,52 \mathrm{a}$ \\
\hline & P 3 & $28,18 \mathrm{~b}$ \\
\hline \multirow{3}{*}{ Profundidade dentro de Roda 1 e Carga 3} & P 1 & $28,88 \mathrm{a}$ \\
\hline & $\mathrm{P} 2$ & $28,50 \mathrm{~b}$ \\
\hline & P 3 & $28,20 \mathrm{c}$ \\
\hline \multirow{3}{*}{ Profundidade dentro de Roda 2 e Carga 1} & P 1 & $29,38 \mathrm{a}$ \\
\hline & $\mathrm{P} 2$ & $29,08 \mathrm{~b}$ \\
\hline & P 3 & $28,58 \mathrm{c}$ \\
\hline \multirow{3}{*}{ Profundidade dentro de Roda 2 e Carga 2} & P 1 & $29,55 \mathrm{a}$ \\
\hline & $\mathrm{P} 2$ & $28,90 \mathrm{~b}$ \\
\hline & P 3 & $28,55 \mathrm{c}$ \\
\hline \multirow{3}{*}{ Profundidade dentro de Roda 2 e Carga 3} & P 1 & $29,52 \mathrm{a}$ \\
\hline & $\mathrm{P} 2$ & $28,70 \mathrm{~b}$ \\
\hline & P 3 & $28,48 \mathrm{c}$ \\
\hline \multirow{3}{*}{ Profundidade dentro de Roda 3 e Carga 1} & P 1 & $28,65 \mathrm{a}$ \\
\hline & $\mathrm{P} 2$ & $28,68 \mathrm{a}$ \\
\hline & P 3 & $28,25 \mathrm{~b}$ \\
\hline \multirow{3}{*}{ Profundidade dentro de Roda 3 e Carga 2} & P 1 & $29,05 \mathrm{a}$ \\
\hline & $\mathrm{P} 2$ & $28,78 \mathrm{~b}$ \\
\hline & P 3 & $28,32 \mathrm{c}$ \\
\hline \multirow{3}{*}{ Profundidade dentro de Roda 3 e Carga 3} & P 1 & $28,80 \mathrm{a}$ \\
\hline & $\mathrm{P} 2$ & $28,85 \mathrm{a}$ \\
\hline & P 3 & $28,50 \mathrm{~b}$ \\
\hline
\end{tabular}

Em cada coluna, para cada fator, médias seguidas de mesma letra não diferem entre si, pelo teste de Tukey a 5\% de probabilidade.

\section{CONCLUSÕES}

As rodas com maior área de contato com o solo proporcionaram maiores valores do teor médio de água e da temperatura do solo.

A temperatura do solo foi menor nas menores profundidades de semeadura e o teor de água do solo não sofreu alterações significativas devido ao fator profundidade. O nível de carga sobre a roda compactadora não afetou a temperatura e o teor de água do solo.

\section{REFERÊNCIAS BIBLIOGRÁFICAS}

AMERICAN SOCIETY OFAGRICULTURAL ENGINEERS. ASAE standard: ASAE S477terminology for soil-engaging components for conservation-tillage planters, drills and seeders. In: . Agricultural engineers yearbook of standards. Saint Joseph, 1995. p. 312-317. 
CORTEZ, J. W.; CARVALHO FILHO, A.; SILVA, R. P.; FURLANI, C. E. A.; SIMONIS, D. B. Comportamento da cultura do sorgo em função do tipo de rodas compactadoras e cargas verticais impostas na operação de semeadura. Engenharia Agrícola, Jaboticabal, v. 26, n. 2, p. 461-469, 2006.

EMPRESA BRASILEIRA DE PESQUISA AGROPECUÁRIA. Centro Nacional de Pesquisa de Solos. Manual de métodos de análise do solo. Rio de Janeiro, 1997. $212 \mathrm{p}$.

EMPRESA BRASILEIRA DE PESQUISA AGROPECUÁRIA. Centro Nacional de Pesquisa dos Solos. Sistema brasileiro de classificação dos solos. Brasília, DF, 1999. $412 \mathrm{p}$.

MONTEIRO, J. A.; CRUZ, J. C.; SANS, L. M. A.; BAHIA, F. G. T. C.; SANTANA, D. P.; GARCIA, J. C.; BAHIA FILHO, A. F. C. Produção de milho no Brasil: realidade e perspectiva. In: CONGRESSO NACIONAL DE MILHO E SORGO, 19., 1992, Porto Alegre. Conferências... Porto Alegre: SAA/SCT/ABMS/EMATER-RS/CNPMSEmbrapa/CIENTEC, 1992. p. 81-126.

PACHECO, E. P. Avaliação de uma semeadora-adubadora de precisão com modificações no tubo condutor de sementes. 1994. 61 f. Dissertação (Mestrado em Engenharia
Agrícola - Mecanização Agrícola) - Universidade Federal de Viçosa, Viçosa, 1994.

POPINIGIS, F. Fisiologia da semente. Brasília, DF: Abrates, 1985. $286 \mathrm{p}$.

RAINBOW, R. Press wheels and their use in seedling. Alexandria: South Australian Research and Development Institute, 2000. Disponível em: <http://www.sardi.sa.gov.au/ crops/farmsys/f_tillag.htm>. Acesso em: 25 jun. 2000.

SILVA, F. M. Influência do tipo de rodas compactadoras de semeadoras-adubadoras no condicionamento físico do solo e no desenvolvimento de plantas. 1990. 131 f. Dissertação (Mestrado em Engenharia Agrícola - Máquinas Agrícolas) - Universidade Estadual de Campinas, Campinas, 1990.

SILVA, R. P.; CORÁ, J. E.; LOPES, A.; FURLANI, C. E. A. Ação de rodas compactadoras de semeadoras submetidas a cargas verticais na deformação do solo. Ciência e Agrotecnologia, Lavras, v. 29, n. 4, p. 839-847, 2005.

SILVA, R. P.; FURLANI, C. E. A.; LOPES, A.; NASCIMENTO, A.; CAMARA, F. T. Efeitos da roda compactadora de semeadoras sob cargas verticais na deformação do solo com dois teores de água. Engenharia Agrícola, Jaboticabal, v. 26, n. 2, p. 511-519, 2006. 